\title{
Effect of a multi-tiered dispatch system on out-of-hospital cardiac arrest patients: preliminary report from the Gyeonggi province, South Korea
}

\author{
Kyung Hune Cho', Jong-Hak Park', Sung Woo Moon ${ }^{1}$, \\ Seong-Keun Yun ${ }^{2}$, Jin-young Kim² \\ 'Department of Emergency Medicine, Korea University Ansan Hospital, Ansan, Korea \\ ${ }^{2}$ Gyeonggi Provincial Fire and Disaster Headquarters, Suwon, Korea
}

Objective In South Korea, the Gyeonggi Fire Services introduced a multi-tiered dispatch system for out-of-hospital cardiac arrest (OHCA) cases in July 2015. In this study, we investigated whether the multi-tiered dispatch system improved the pre-hospital return of the spontaneous circulation (ROSC) rate.

Methods All non-traumatic adult OHCAs treated and transported by the 119 emergency medical system from July 2015 to December 2015 were included in the study. Demographic and pre-hospital Utstein element-data were collected from the emergency medical system OHCA database. The primary outcome was pre-hospital ROSC as measured at the scene.

Results Of the included OHCAs, 1,436 (89.0\%) were categorized to the single-tiered dispatch group and $162(10.1 \%)$ to the multi-tiered dispatch group. The rate of administration of advanced airway ventilation (61.1\% vs. $48.0 \%, P=0.002)$ and intravenous access ( $18.5 \%$ vs. $12.5 \%$, $\mathrm{P}=0.037)$ was higher in the multi-tiered group compared to that in the single-tiered group. The use of epinephrine was higher in the multi-tiered group ( $4.9 \%$ vs. $1.5 \%, \mathrm{P}=0.002)$. The pre-hospital ROSC rates in the multi-tiered group were higher when compared with the single-tiered group, but the difference was not significant (10.5\% vs. $7.5 \%, \mathrm{P}=0.218)$. The adjusted odds ratio for pre-hospital ROSC rates in the multi-tiered group was 1.29 (95\% confidence interval, 0.69 to 2.40).

Conclusion The multi-tiered dispatch system was not associated with a significant increase in the pre-hospital ROSC rate during the early phase of its implementation, even though advanced maneuvers were performed more frequently.

Keywords Emergency medical service; Out-of-hospital cardiac arrest; Korea
eISSN: 2383-4625

Received: 25 May 2017

Revised: 8 October 2017

Accepted: 16 October 2017

Correspondence to: Jong-Hak Park Department of Emergency Medicine, Korea University Ansan Hospital, 23 Jeokgeum-ro, Danwon-gu, Ansan 15355, Korea

E-mail: rosc@hanmail.net

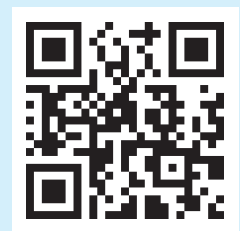

How to cite this article:

Cho KH, Park JH, Moon SW, Yun SK, Kim J. Effect of a multi-tiered dispatch system on out-of-hospital cardiac arrest patients: preliminary report from the Gyeonggi province, South Korea. Clin Exp Emerg Med 2018;5(3):144-149.
This is an Open Access article distributed under the terms of the Creative Commons Attribution Non-Commercial License (http:// creativecommons.org/licenses/by-nc/4.0/). 


Wapsule $\begin{aligned} & \text { What is already known } \\ & \text { In South Korea, there have been limitations on performing optimal cardiopulmonary resuscitation because the } 119 \text { emer- } \\ & \text { gency medical system (EMS) system deploys only } 2 \text { to } 3 \text { emergency medical technicians per ambulance. } \\ & \text { What is new in the current study } \\ & \text { The present preliminary study suggests that the multi-tiered dispatch system with larger number of EMS personnel on } \\ & \text { the scene was not associated with a significant increase in the pre-hospital return of the spontaneous circulation rate } \\ & \text { for the intermediate-level EMS system in South Korea, when compared to the single-tiered dispatch system. }\end{aligned}$

\section{INTRODUCTION}

In South Korea, approximately 30,000 cases of out-of-hospital cardiac arrest (OHCA) occur each year. The overall survival rate was 5.1\% in 2014. ${ }^{1}$ With early and effective resuscitation, OHCA is treatable, and the quality of cardiopulmonary resuscitation (CPR) is closely linked to outcomes. ${ }^{2-7}$ Individual communities have attempted to improve outcomes by systematically improving CPR quality delivered by out-of-hospital providers, and several have reported significantly higher survival rates., ${ }^{3,8-13}$

In South Korea, there are certain limitations to improving CPR quality on the scene. We believe that one such limitation is that the emergency medical service (EMS) system deploys 2 to 3 emergency medical technicians (EMTs) per ambulance, and the small number of EMTs at the scene affects CPR quality. To overcome this issue, the Gyeonggi province fire services implemented a multitiered dispatch system for OHCA and severe trauma patients, but the effectiveness of multi-tiered dispatch is still questionable.

The aim of this pilot study was to determine whether the multitiered dispatch system for non-traumatic adult OHCA patients with presumed cardiac etiology improved pre-hospital return of the spontaneous circulation (ROSC) rates. We also assessed the pre-hospital activity of EMS providers in the multi-tiered dispatch system.

\section{METHODS}

\section{Study design and setting}

The study was a retrospective, cross-sectional preliminary study of non-traumatic adult OHCAs in the Gyeonggi province, South Korea.

The Gyeonggi province of South Korea has an area of 10,172 $\mathrm{km}^{2}$, and a population of $12,340,000$, as estimated in 2014. In 2015 , the Gyeonggi province fire service recorded 382,217 cases of ambulance transport, and of these, cardiac arrests accounted for 6,379 cases. There was a total of 222 functioning ambulances with 1,184 EMTs on active duty, including 799 level 1 EMTs and nurses. ${ }^{14} \mathrm{~A}$ typical responding ambulance crew consists of 2 to 3 persons. The quality of EMS service in the Gyeonggi province is equivalent to that of intermediate-level service in North America. ${ }^{15}$ The EMS personnel provide CPR on-scene according to the 2010 American Heart Association CPR guidelines, with a 30:2 compression-to-ventilation ratio, and advanced airway or bagvalve mask ventilation. The law in South Korea does not routinely allow EMTs to use resuscitation drugs, such as epinephrine at the scene, though use as per direct medical direction from a doctor is allowed. ${ }^{15,16}$ The EMTs are encouraged to stay and provide CPR for at least 5 minutes at the scene. ${ }^{16}$ In addition, there were a total of 229 fire engines equipped with an automated external defibrillator with firefighters trained in providing basic life support. ${ }^{14}$ Fire engines responded to treat emergent patients while an ambulance was en route. A typical fire engine crew consists of 3 to 4 fire fighters.

Until May 2015, the Gyeonggi province fire services employed a single-tiered system that deployed a single ambulance to respond to any EMS call. Beginning July 2015, the Gyeonggi province fire services implemented a multi-tiered system that deploys 2 ambulances or 1 ambulance and 1 fire engine to the scene when possible, for cases of cardiac arrest or suspected severe trauma. This service is active in 22 cities: Seongnam city, Bucheon city, Ansan city, Anyang city, Namyangju city, Uijungbu city, Siheung city, Gwangmyeong city, Paju city, Gunpo city, Gwangju city, Gimpo city, Icheon city, Yangju city, Uiwang city, Hanam city, Pocheon city, Dongducheon city, Gwacheon city, Yeoju city, Gapyeong county, and Yeoncheon county.

For suspected cardiac arrest situations, multi-tiered dispatch was selected by a dispatcher at the Gyeonggi Fire Headquarters when the required ambulances or fire engines were available in close proximity. When the dispatcher did not recognize cardiac arrest, but the EMS personnel in transit suspected cardiac arrest, 
the EMS personnel requested an additional ambulance or fire engine from the headquarters.

\section{Participants and data collection}

The study period extended from July 2015 (when the multi-tiered dispatch system was newly implemented in selected areas for OHCAs in the Gyeonggi province) to December 2015. The OHCAs which occurred in regions where other tiered EMS systems were in effect were excluded. All patients over 18 years of age who suffered non-traumatic OHCA with presumed cardiac etiology and who were treated and transported to the hospital by the 119 EMS were included in the study. Enrolled patients were categorized into either multi-tiered or single-tiered dispatch groups. We used the 119 EMS OHCA database (EMS rescue run sheets and EMS cardiac arrest registry) to extract data for demographic and pre-hospital Utstein elements. The variables collected were: type of dispatch, age, sex, location of arrest, witnessed versus unwitnessed arrest, provision of bystander CPR, initial cardiac rhythm on EMS arrival, defibrillation by 119 EMTs, provision of advanced airway ventilation, intravenous access, resuscitation drug use, time from EMS dispatch to on-scene arrival (response interval), scene time interval, and pre-hospital ROSC.

We defined single-tired dispatch as that managed by provision of a single ambulance and multi-tiered dispatch as that managed by provision of two or more vehicles (2 ambulances or 1 ambulance and 1 fire engine). Pre-hospital ROSC was defined as any ROSC sustained at the scene with spontaneous circulation maintained until arrival at the hospital.

\section{Data analysis and statistics}

The primary outcome was pre-hospital ROSC as measured at the scene by 119 EMTs. The secondary outcome was the activity of 119 EMTs during resuscitation at the scene. These outcomes were compared between the single and multi-tiered dispatch groups.

Continuous variables are presented as means and standard deviations. Categorical variables are reported as frequencies with percentages. The Student t-test for continuous variables was used for comparisons between the two groups, and the chi-squared test was used for categorical variables. Multivariable logistic regression analysis was used to compare primary outcomes between the groups and to calculate the adjusted odds ratios at 95\% confidence intervals. Age, sex, initial rhythm, location of arrest, witness status, provision of bystander $\mathrm{CPR}$, and on-scene time were included as co-variables. Data were analyzed using IBM SPSS Statistics ver. 20 (IBM Corp., Armonk, NY, USA). A P-value < 0.05 was considered to indicate statistical significance.

\section{Ethical statement}

The institutional review board of Korea University Ansan Hospital for clinical research approved the use of 119 EMS OHCA records for this retrospective study (AS16180). The informed consent was waived by the board.

\section{RESULTS}

There were a total of 5,602 EMS-assessed OHCAs in the Gyeonggi province during the study period. Of these, 4,001 were excluded, and the remaining 1,601 adult cases of presumed cardiac etiology

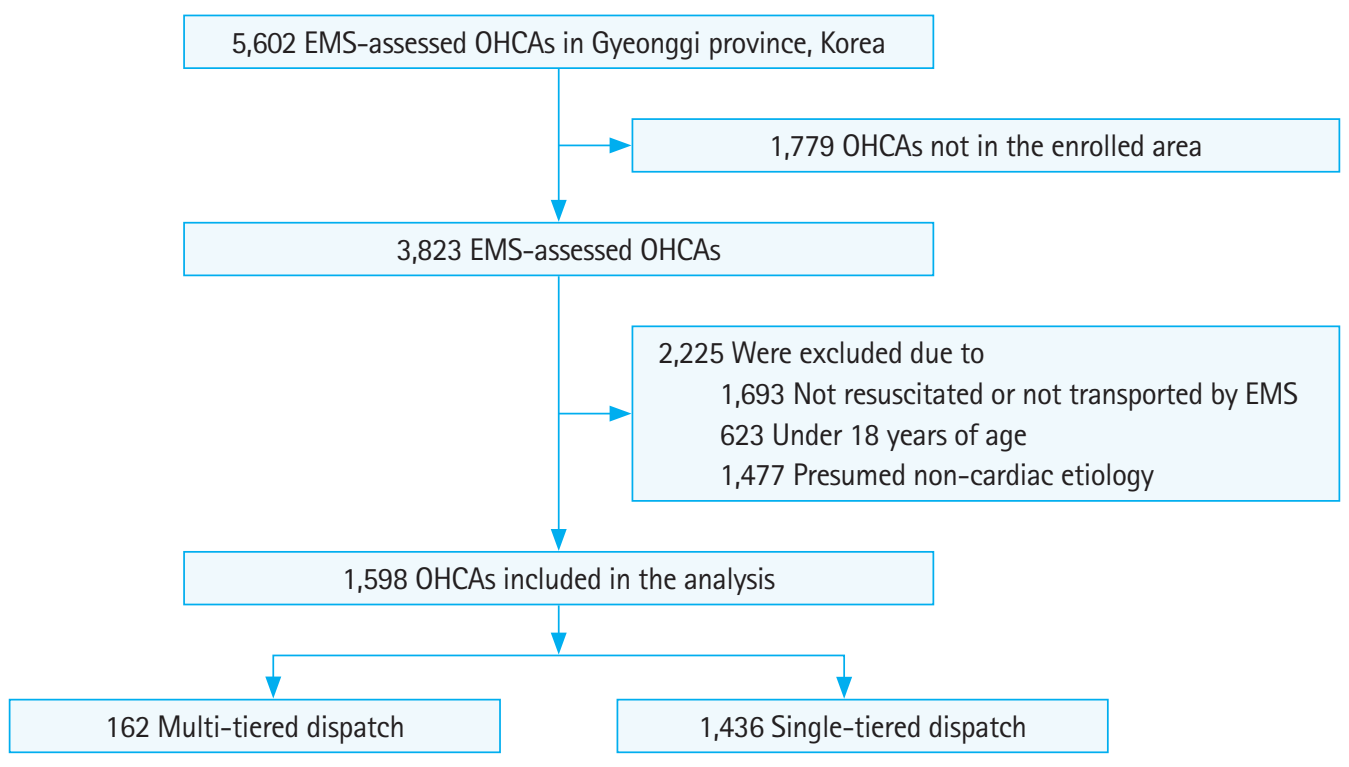

Fig. 1. Flowchart of study enrollment. EMS, emergency medical service; OHCA, out-of-hospital cardiac arrest. 
that underwent resuscitation and transportation to the hospital by 119 EMS were included in the analysis (Fig. 1). Of the included OHCAs, 1,436 (89.0\%) were assigned to the single-tiered dispatch group and 162 (10.1\%) to the multi-tiered dispatch group.

Patient characteristics and variable-associated outcomes are presented in Tables 1 and 2, respectively, along with comparisons between the groups. The two groups did not differ significantly in age, sex, initial arrest rhythm, location of arrest, or witness status. Bystander CPR was significantly more frequent in the multi-tiered group $(71.9 \%$ vs. $65.7 \%, \mathrm{P}<0.001)$. Mean response time was $9.0 \pm 5.7$ minutes in the multi-tiered group, and $8.3 \pm 4.5$ minutes in the single-tiered group $(P=0.146)$. Mean scene-time intervals were $13.0 \pm 6.1$ minutes in the multi-tiered group, and $12.9 \pm 6.2$ minutes in the single-tiered group $(P=0.831)$. Advanced airway ventilation was secured in 99 patients $(61.1 \%)$ in the multi-tiered group and in $689(48.0 \%)$ patients in the single-tiered group $(\mathrm{P}=$ 0.002). Intravenous access was obtained in 30 patients $(18.5 \%)$ in the multi-tiered group, and in 180 patients (12.5\%) in the single-tiered group $(P=0.037)$. The use of epinephrine was more frequent in the multi-tiered group than in the single-tiered group $(P=0.002)$. Pre-hospital ROSC was higher in the multi-tiered group compared to that in the single-tiered group, though the difference was not statistically significant ( $10.5 \%$ vs. $7.5 \%, P=0.218)$.

Table 1. Comparison of the characteristics of enrolled patients between dispatch groups

\begin{tabular}{lcccc}
\hline & $\begin{array}{c}\text { Total } \\
(n=1,598)\end{array}$ & $\begin{array}{c}\text { Multi-tiered Single-tiered } \\
\text { dispatch } \\
(n=162)\end{array}$ & $\begin{array}{c}\text { dispatch } \\
(n=1,436)\end{array}$ & P-value \\
& $67.75 \pm 15.5$ & $66.8 \pm 15.2$ & $68.7 \pm 15.8$ & 0.137 \\
\hline Age (yr) & $1,038(65.0)$ & $120(74.1)$ & $918(63.8)$ & 0.009 \\
Sex, male & $767(48.0)$ & $72(44.4)$ & $695(48.3)$ & 0.363 \\
Witnessed arrest & $1,011(63.3)$ & $123(75.9)$ & $888(61.7)$ & $<0.001$ \\
Provision of bystander CPR & & & & \\
Initial cardiac rhythm & $258(16.1)$ & $30(18.5)$ & $228(15.8)$ & 0.369 \\
$\quad$ Shockable & $1,343(84.0)$ & $132(81.5)$ & $1,211(84.2)$ & \\
$\quad$ Non-shockable & & & & \\
Location of arrest & $1,015(63.5)$ & $98(60.5)$ & $917(63.7)$ & 0.439 \\
$\quad$ Home & $8.3 \pm 4.6$ & $9.0 \pm 5.7$ & $8.3 \pm 4.5$ & 0.146 \\
Response time (min) & $11.5 \pm 5.7$ & $11.9 \pm 6.0$ & $11.4 .9 \pm 5.7$ & 0.289 \\
On-scene time (min) & & & & \\
\hline
\end{tabular}

Values are presented as mean \pm standard deviation or number (\%). $\mathrm{CPR}$, cardiopulmonary resuscitation.
Adjusted odds ratios for pre-hospital ROSC rate in the multi-tiered group compared to that in the single-tiered group using multivariable logistic regression was 1.29 (95\% confidence interval, 0.69 to 2.40 ) (Table 3).

\section{DISCUSSION}

The present preliminary study suggests that the multi-tiered dispatch system, during the early phase of its implementation, did not facilitate a significant increase in the pre-hospital ROSC rate for the intermediate-level EMS system in South Korea when compared to the single-tiered dispatch system, even though certain advanced maneuvers were performed more frequently at the scene.

Multiple factors affect the survival of OHCA patients, with one possible component being the quality of CPR provided at the scene. ${ }^{3,9,10,17,18}$ High-quality CPR results in higher coronary and cerebral perfusion pressures and is associated with improved survival and neurologic outcomes. The EMS providers in South Korea generally perform sub-optimal quality CPR, largely because of the small number of EMS personnel deployed. With a restricted number of deployed personnel on-scene, consisting of only 2 to 3 persons, there are definite limitations to the quality of CPR that can be provided. To overcome this problem, the multi-tiered system that deploys 2 ambulances or 1 ambulance and 1 fire engine to the scene was implemented for cardiac arrest and severe trauma patients in the Gyeonggi province.

A larger number of EMS personnel on the scene, through the multi-tiered dispatch system, can help maintain proper compression rate, depth, and duty cycle, as well as limit interruptions. Ad-

Table 2. Variables associated with EMTs activity at the scene and outcome

\begin{tabular}{lccc}
\hline & $\begin{array}{c}\text { Multi-tiered dis- } \\
\text { patch }(n=162)\end{array}$ & $\begin{array}{c}\text { Single-tiered dis- } \\
\text { patch }(n=1,436)\end{array}$ & P-value \\
\hline Defibrillation by EMTs & $43(26.5)$ & $314(21.9)$ & 0.195 \\
Advanced airway insertion & $99(61.1)$ & $689(48.0)$ & 0.002 \\
Intravenous access & $30(18.5)$ & $180(12.5)$ & 0.037 \\
Use of epinephrine & $8(4.9)$ & $22(1.5)$ & 0.002 \\
Pre-hospital ROSC & $17(10.5)$ & $110(7.7)$ & 0.218 \\
\hline
\end{tabular}

Values are presented as number (\%).

EMT, emergency medical technician; ROSC, return of spontaneous circulation.

Table 3. Multivariable logistic regression for pre-hospital return of the spontaneous circulation

\begin{tabular}{lcccccc}
\hline & Number & $\%$ & OR & $95 \% \mathrm{Cl}$ & aOR & 95\% Cl \\
\hline Single-tiered dispatch group (reference) & 110 & 86.6 & Reference & - & Reference & - \\
Multi-tiered dispatch group & 17 & 13.4 & 1.42 & $0.83-2.43$ & 1.29 & $0.69-2.40$ \\
\hline
\end{tabular}

Adjusted co-variables: sex, age, initial cardiac rhythm, location of arrest, witness status, bystander cardiopulmonary resuscitation, response time, and on-scene time. $\mathrm{OR}$, odds ratio; $\mathrm{Cl}$, confidence interval; $\mathrm{aOR}$, adjusted odds ratio. 
ditionally, this system also limits the amount of time a rescuer performs compressions, and leads to lower fatigue. The teambased approach, wherein pre-hospital responders perform a choreographed role in resuscitation, also leads to higher-quality CPR and patient care. Previous studies suggest higher survival rates among patients who received team-based CPR. ${ }^{2,12,13}$

Another advantage that can be expected from multi-tiered dispatch is that time from arrest to first chest compression by trained EMS personnel can be shortened. This shortened interval is likely to be advantageous in situations where an ambulance is not available near the scene, or if the fire engine arrives earlier than the ambulance. Trained EMS personnel may perform CPR in a timelier manner compared to laypersons, and this will likely have a positive impact on pre-hospital ROSC rates.

However, during the study period, there was no increase in pre-hospital ROSC rates in the multi-tiered dispatch group. There are several possible reasons for this result. One reason is that the study period may be too short to adequately reflect the impact of the multi-tiered system. Over the 6-month study period, multitiered dispatch occurred for about 10\% of included patients. This low figure may be because of deficiencies in dispatcher recognition of cases of cardiac arrest and insufficient experience in directing multi-tiered dispatch.

Another reason is that it is likely that official education or handson training was lacking prior to deployment. Well-trained EMTs can guarantee higher-quality emergency medical treatment in the pre-hospital phase. Nevertheless, for high-quality CPR, it is necessary that each member of the team carries out a specific role, in conjunction with their teammates. Efficient CPR might not have been performed in the early phase because of the absence of such educational programs, although there were many EMTs at the scene. Scene-times were not greater in the multi-tiered group for the same reason, even though intravenous access, drug use, and advanced airway ventilation deployment rates were higher in the multi-tiered dispatch group.

This study has several limitations. First, the primary outcome only measures pre-hospital ROSC, and does not consider the final outcome of the patient after in-hospital care. Pre-hospital ROSC rate alone may not have been sufficient to determine an improvement in EMS resuscitative care. However, ROSC at the scene is more closely related to the survival of cardiac arrest patients compared to other factors. ${ }^{19,20}$ Second, CPR quality itself, such as compression depth or release velocity, was not measured in this study. Third, as the multi-tiered system was rolled out in certain select areas, a relatively small number of actual multi-tiered deployments were available for analysis. Another limitation is selection bias, originating from the dispatcher selecting the type of dispatch. We believe that the selection bias was minimal, as the dispatch type was not decided according to the patients but the situation of the area. Finally, the study period of 6 months may also have been too short to collect a critical mass of data sufficiently large enough to reflect the true impact of the multi-tiered system. Despite these shortcomings, this study has value as a pilot study, and can serve as a guide to further study of the EMS dispatch system in South Korea.

In conclusion, the present retrospective preliminary study suggests that a multi-tiered dispatch system did not affect a significant increase in the pre-hospital ROSC rate during the early phase of its implementation, even though advanced maneuvers were performed more frequently. Given these results, further study is needed after implementation of ongoing improvement efforts, including education for team-based CPR as well as system-monitoring and feedback.

\section{CONFLICT OF INTEREST}

No potential conflict of interest relevant to this article was reported.

\section{REFERENCES}

1. Korea Centers for Disease Control and Prevention. The 3rd International Symposium of Acute Cardiac Arrest Survey. Cheongju: Korea Centers for Disease Control and Prevention; 2014.

2. Weng $\mathrm{Tl}$, Huang $\mathrm{CH}, \mathrm{Ma} \mathrm{MH}$, et al. Improving the rate of return of spontaneous circulation for out-of-hospital cardiac arrests with a formal, structured emergency resuscitation team. Resuscitation 2004;60:137-42.

3. Lund-Kordahl I, Olasveengen TM, Lorem T, Samdal M, Wik L, Sunde K. Improving outcome after out-of-hospital cardiac arrest by strengthening weak links of the local Chain of Survival; quality of advanced life support and post-resuscitation care. Resuscitation 2010;81:422-6.

4. Reynolds JC, Bond MC, Shaikh S. Cardiopulmonary resuscitation update. Emerg Med Clin North Am 2012;30:35-49.

5. Bobrow BJ, Spaite DW, McNally BF. Cardiac arrest registry to enhance survival to begin collecting data to measure CPR quality. JEMS 2015;40:56-63.

6. Meaney PA, Bobrow BJ, Mancini ME, et al. Cardiopulmonary resuscitation quality: [corrected] improving cardiac resuscitation outcomes both inside and outside the hospital: a consensus statement from the American Heart Association. Circulation 2013;128:417-35.

7. Hasselqvist-Ax I, Riva G, Herlitz J, et al. Early cardiopulmonary 
resuscitation in out-of-hospital cardiac arrest. N Engl J Med 2015;372:2307-15.

8. Stefos KA, Nable JV. Implementation of a high-performance cardiopulmonary resuscitation protocol at a collegiate emergency medical services program. J Am Coll Health 2016;64: 329-33.

9. Nichol G, Leroux B, Wang $H$, et al. Trial of continuous or interrupted chest compressions during CPR. N Engl J Med 2015; 373:2203-14.

10. Bobrow BJ, Clark LL, Ewy GA, et al. Minimally interrupted cardiac resuscitation by emergency medical services for out-ofhospital cardiac arrest. JAMA 2008;299:1158-65.

11. Rea TD, Page RL. Community approaches to improve resuscitation after out-of-hospital sudden cardiac arrest. Circulation 2010;121:1134-40.

12. Stopyra JP, Courage $C$, Davis CA, Hiestand BC, Nelson RD, Winslow JE. Impact of a "Team-focused CPR" protocol on out-ofhospital cardiac arrest survival in a rural EMS system. Crit Pathw Cardiol 2016;15:98-102.

13. Pearson DA, Darrell Nelson $R$, Monk $L$, et al. Comparison of team-focused CPR vs standard CPR in resuscitation from outof-hospital cardiac arrest: results from a statewide quality improvement initiative. Resuscitation 2016;105:165-72.

14. Ministry of Public Safety and Security. Statistical yearbook
2015 of 119 emergency services. Sejong: Ministry of Public Safety and Security; 2016.

15. Ministry of Government Legislation. The law about emergency medical care. No. 42: partial revision. Seoul: Ministry of Health and Welfare; 2011.

16. Ministry of Public Safety and Security. The standard protocol for 119 emergency medical services providers. Sejong: Ministry of Public Safety and Security; 2014.

17. Wik L, Kramer-Johansen J, Myklebust H, et al. Quality of cardiopulmonary resuscitation during out-of-hospital cardiac arrest. JAMA 2005;293:299-304.

18. Bobrow BJ, Vadeboncoeur TF, Stolz $U$, et al. The influence of scenario-based training and real-time audiovisual feedback on out-of-hospital cardiopulmonary resuscitation quality and survival from out-of-hospital cardiac arrest. Ann Emerg Med 2013;62:47-56.

19. Wampler DA, Collett $L$, Manifold CA, Velasquez C, McMullan JT. Cardiac arrest survival is rare without prehospital return of spontaneous circulation. Prehosp Emerg Care 2012;16:451-5.

20. Drennan IR, Lin S, Sidalak DE, Morrison $\amalg$. Survival rates in out-of-hospital cardiac arrest patients transported without prehospital return of spontaneous circulation: an observational cohort study. Resuscitation 2014;85:1488-93. 